\title{
Geburt im Rettungsdienst - Das sollten Sie wissen für die Ergänzungsprüfung
}

Rico Kuhnke, Wolfgang von Meißner

\section{Fallbeispiel}

\section{Einsatzmeldung}

Sonntagvormittag. Die Besatzung des Rettungswagens ist auf dem Weg zu einer bevorstehenden Geburt. Die Einsatzmeldung der Leitstelle lautet: „Patientin in der 38. Schwangerschaftswoche, beginnende Wehentätigkeit, liegender Transport in die geburtshilfliche Abteilung des Kreiskrankenhauses."
Vor dem Einfamilienhaus werden Sie bereits von dem aufgeregt winkenden Ehemann erwartet. Dieser schildert, die Fruchtblase sei mittlerweile geplatzt und seine Frau habe den Eindruck, die Geburt stehe kurz bevor. Das Team entscheidet, neben dem Standardequipment auch den Kindernotfallrucksack mit Geburtsset mitzunehmen.

\section{Lagebeurteilung}

Die Ehefrau sitzt im Bademantel im Wohnzimmer auf dem Sofa. Die Wehen scheinen im Augenblick etwas 
nachgelassen zu haben. Die Kollegin notiert sich die Zeit. Das Gesicht der Kreißenden wirkt entspannt.

Die werdende Mutter schildert dem Team kurz die Situation: Die Wehen hätten vor rund zwei Stunden mit einem Ziehen im Rücken begonnen. Da die Geburt sich beim letzten Mal über 10 Stunden hingezogen habe, habe sie sich in aller Ruhe bereitgemacht und sich in der Klinik angemeldet. Diese habe wegen des Fruchtwasserabgangs den Rettungswagen verständigt. Ihren dreijährigen Sohn habe die Schwiegermutter bereits vor einigen Stunden abgeholt.

Während der Schilderungen setzt die Wehentätigkeit erneut ein. Ein Blick auf die Uhr zeigt, dass seit dem Eintreffen knapp 2 Minuten vergangen sind. Der Notfallsanitäter legt seine Hand sanft auf den Unterarm der Kreißenden und tastet dabei den Puls an der A. radialis.

\section{Bevorstehende Geburt}

Während er den Puls tastet, leitet er die Patientin dazu an, die einsetzenden Presswehen durch gleichmäßige, tiefe Atemzüge zu lindern. Der Puls ist regelmäßig und gut tastbar, die Frequenz mit 120 Schlägen pro Minute tachykard.

Aufgrund der Schilderungen der Frau und der regelmäßigen Wehentätigkeit rechnet das Team mit einer bevorstehenden Geburt ( $\triangleright$ Abb. 1). Während sich die Kollegin um die Betreuung der Kreißenden kümmert und sich den Mutterpass geben lässt, hat ihr Kollege im Wohnzimmer Platz geschaffen, die Heizung hochgedreht und auf dem Boden alles für die Geburt vorbereitet. Auf mehreren Lagen frischer Badetücher hat er ein Einmallaken ausgebreitet. Im Bereich des Beckens liegt zusätzlich eine saugstarke Unterlage.

Die Gebärende wird gebeten, sich auf die am Boden ausgebreiteten weichen Badetücher zu setzen. Ihr Partner kniet sich hinter sie und stützt ihren Oberkörper in leicht erhöhter Position. Um es der Frau zusätzlich bequem zu machen, wird der Rücken durch ein Kissen gepolstert.

\section{Informationen aus dem Mutterpass}

Die Frau ist zweitgebärend; die erste Geburt verlief ohne Komplikationen. Auch die aktuelle Schwangerschaft gibt keinen Hinweis auf Schwierigkeiten; die letzte Eintragung der Frauenärztin stammt vom Vortag, und die festgestellte Schädellage des Kindes bestärkt das Team in der Entscheidung, die Geburt vor Ort abzuwarten.

\section{Zusammenfassung der Untersuchungs- ergebnisse}

Während einer Pause zwischen den Wehen bespricht sich das Team des Rettungswagens kurz und fasst die Untersuchungsergebnisse strukturiert zusammen.
A-irway

Die Atemwege sind frei. Deutlich sichtbar hebt und senkt sich der Brustkorb der Gebärenden. Die Frau ist wach und voll orientiert.

\section{B-reathing}

Die Atemfrequenz variiert zwischen 15 Atemzügen pro Minute in den Wehenpausen und bis zu 30 Atemzügen während der Presswehen. Die Atmung ist regelmäßig und ausreichend tief. Der angelegte Fingerclip des Pulsoxymeters zeigt einen $\mathrm{SpO}_{2}$ von $98 \%$.

\section{C-irculation}

Der periphere Puls lässt sich an der A. radialis gut tasten. Die Frequenz ist mit 120 Schlägen pro Minute zwar tachykard, aber den Umständen angemessen. Dies gilt auch für den Blutdruck von 115/70 mm Hg.

D-isability

Die Gebärende ist wach und voll orientiert. Auf neurologische Defizite gibt es keinen Hinweis.

\section{E-xposure}

Mittlerweile war die Kollegin der Frau behilflich, den Slip auszuziehen. Der Anus ist weit vorgewölbt und auch die Vulva der Patientin ist bereist leicht gedehnt. Das Team rechnet jederzeit mit der Geburt.

\section{SAMPLER-Schema}

Die Vervollständigung der Anamnese mithilfe des SAMPLER-Schemas ergibt keinen auffälligen Befund.

PRAXIS/MASSNAHMEN

Gängige Merkhilfen haben wir für Sie übersichtlich zusammengefasst. Bestellen Sie Ihren kostenfreien Lernspicker unter www.thieme.de/retten-lernspicker.

\section{Aufklärung der Kreißenden}

Die Kollegin wirkt weiterhin beruhigend auf die Kreißende und den werdenden Vater ein. Dabei erläutert sie, dass die Geburt vor Ort durch das Rettungsdienstpersonal begleitet werden soll. Ihr Kollege habe bereits alles vorbereitet und werde ihr zur Sicherheit nun einen venösen Zugang legen und eine Infusion anschließen.

Da die Frau bereits ein Kind geboren hat und während der Schwangerschaft regelmäßig an Geburtsvorbereitungsseminaren teilgenommen hat, ist nur wenig Anleitung durch das Rettungsdienstpersonal erforderlich. Mittlerweile ist der kindliche Schädel in der Vulva deutlich auszumachen ( $\triangleright$ Abb. 2). 


\section{ABCDE-Herangehensweise}

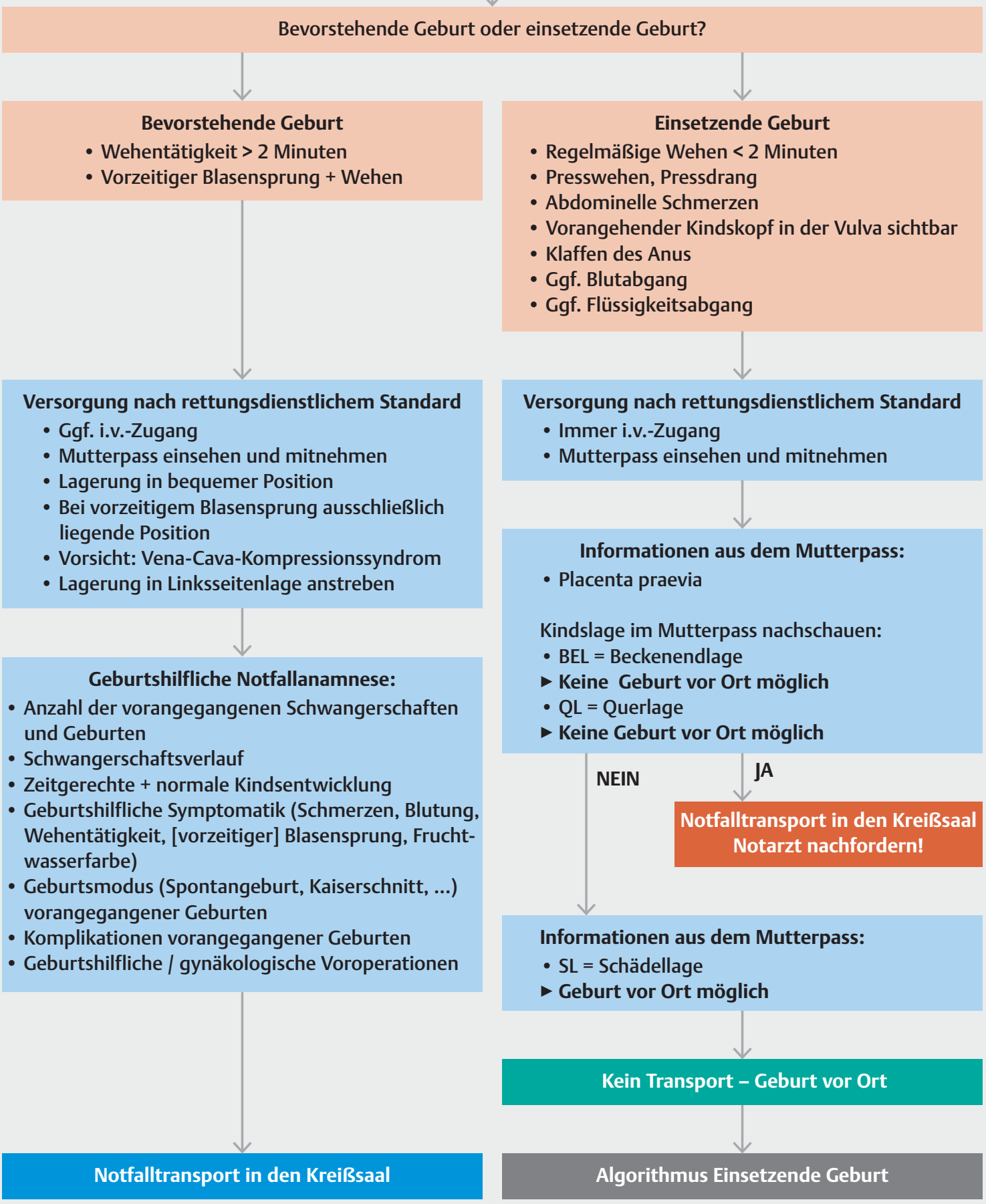

- Abb.1 Algorithmus Bevorstehende Geburt - Transportentscheidung. Quelle: Deutscher Berufsverband Rettungsdienst e. V. 


\section{Einsetzende Geburt}

- Regelmäßige Wehen < 2 MInuten

- Presswehen, Pressdrang

- Abdominelle Schmerzen

- Vorangehender Kindskopf in der Vulva sichtbar

- Klaffen des Anus

- Ggf. Blutabgang

- Ggf. Flüssigkeitsabgang, Blasensprung

- IMMER Anlage i.v.-Zugang

- Ruhige Umgebung schaffen

- Ausreichende Raumtemperatur sicherstellen

- Gebärende und deren Partner beruhigen

- Gebärende nicht mehr laufen lassen

- Lagerung ggf. in Rückenlage mit leicht erhöhtem Oberkörper und angewinkelten, gespreizten Beinen
- Vorsicht: Vena-Cava-Kompressionssyndrom

- Lagerung in Linksseitenlage anstreben

- Sterile Unterlage vorlegen (z. B. Brandwundenverbandtuch)

- Abnabelung vorbereiten:

2 Nabelklemmen, 1 sterile Schere / Skalpell, sterile Kompressen

GEBURT - Geburtsfortschritt beobachten - NICHT EINGREIFEN!

1. Geburt des führenden Kindsteils (meist Kopf!)

- Dammschutz und Führung bei Durchgleiten des Kopfes

- Nach Entwicklung des Kopfes sofortiger Ausschluss einer Umschlingung der Nabelschnur um den Kopf

2. Geburt des kindlichen Körpers

- Vordere Schulter unter Senkung des Kopfes entwickeln, bis die Oberammitte sichtbar ist

- Hintere Schulter unter Anheben des Kopfes OHNE Zug entwickeln

Abnabelung

- Abnabelung erst nach einer Minute (bei akuter Bedrohung sofort!)

- Ca. $20 \mathrm{~cm}$ vom Kind entfernt zwei Nabelklemmen im Abstand von $3 \mathrm{~cm}$ setzen und zwischen den Nabelklemmen durchtrennen

- Abtrocknen des Kindes und Wärmeerhalt mit Tüchern

- Uhrzeit notieren (Abnabelungszeit = Geburtszeit)

- APGAR nach 1 Minute, 5 Minuten und 10 Minuten erheben

- Nachgeburt nicht abwarten - Mutter + Kind (+ ggf. Plazenta) in die nächstgelegene Geburtsklinik transportieren

\section{Algorithmus Neugeborenenversorgung direkt nach der Geburt}

Abb.2 Algorithmus Einsetzende Geburt. Quelle: Deutscher Berufsverband Rettungsdienst.

\section{ZUSATZINFO}

Unterstützen Sie uns!

Für die Rubrik „Fit für den Notfallsanitäter“ verwenden wir die unterschiedlichsten Algorithmen und SOPs aus dem gesamten Bundesgebiet. Deshalb würden wir an dieser Stelle gern auch einen Ihrer Algorithmen vorstellen. Senden Sie uns Ihre regionalen Algorithmen, Handlungsanweisungen und SOPs an retten@thieme.de.

\section{Unterstützung bei der Geburt}

Als sich die nächste Wehe ankündigt, wird die Patientin aufgefordert, dem Pressdrang nachzugeben und mit der Wehe mitzuschieben. Die Kollegin schützt den Damm mit der linken Hand und reguliert mit der anderen Hand die Durchtrittgeschwindigkeit des kindlichen Kopfes ( Abb. 3a). Der Kopf durchtritt nun mit Blick in Richtung 

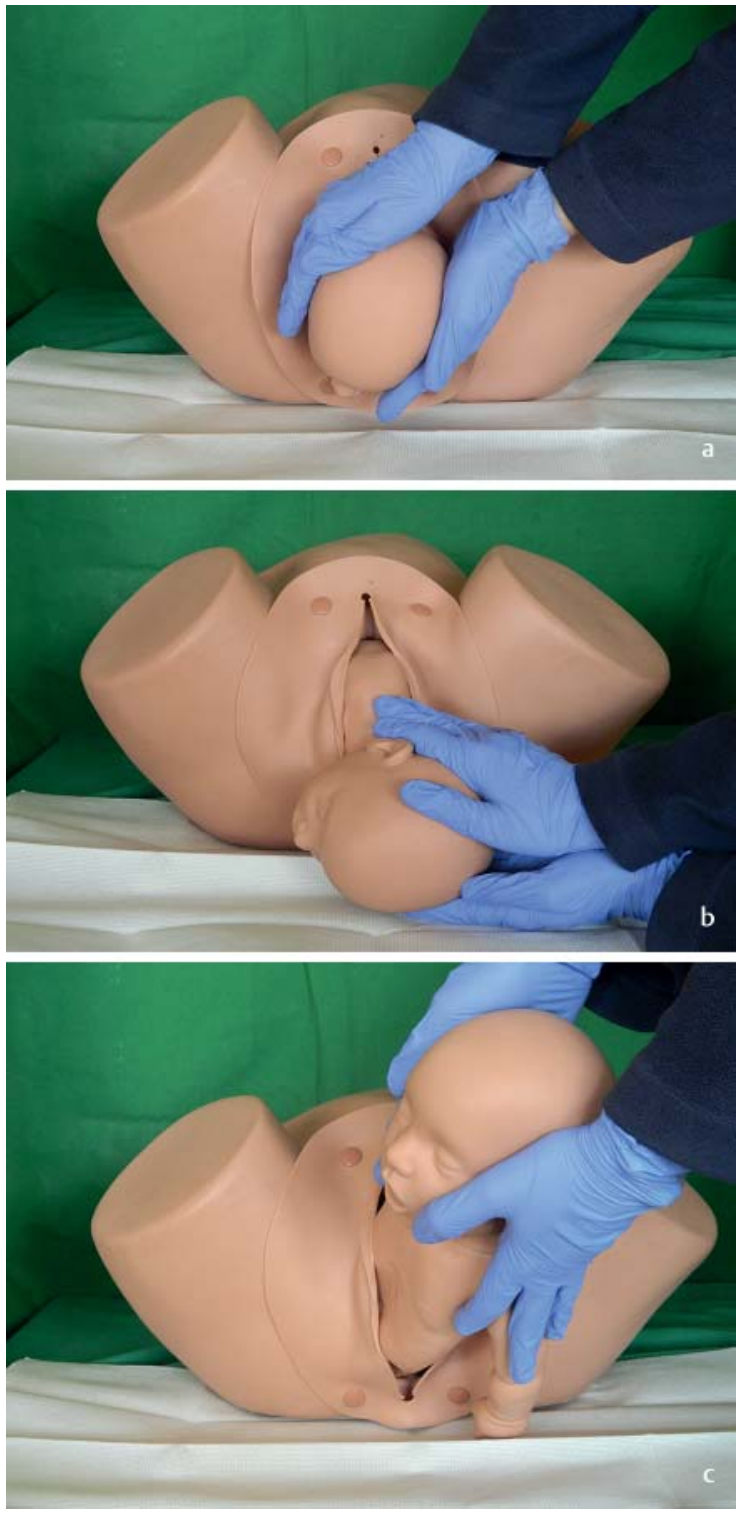

Abb. 3 a Durchtritt des kindlichen Kopfes. b Entwicklung der vorderen Schulter. c Entwicklung der hinteren Schulter.

Anus der Mutter den Beckenausgang. Sanft unterstützt die Kollegin das seitliche Drehen des Kopfes.

Nun wird die Patientin angeleitet, nicht mehr zu pressen und die Wehen durch langsame und tiefe Atemzüge zu verhecheln. Die Notfallsanitäterin sucht den Blickkontakt zur Gebärenden und lächelt ihr aufmunternd zu: „Der Kopf ist bereits geboren und das Schlimmste geschafft. Wir machen eine kleine Pause und mit der nächsten Presswehe kommt das Kleine zur Welt.“

\section{Geburt des kindlichen Körpers}

Mit Beginn der nächsten Wehe führt die Kollegin den Kopf des Kindes sanft nach unten und entwickelt die vordere Schulter ( $>$ Abb. $\mathbf{3 b}$ ). Nachdem der Oberarm bis zur
Mitte sichtbar ist, wird die Gebärende noch einmal aufgefordert mitzupressen. Nun geht alles sehr schnell: Durch Anheben des Kopfes entwickelt sich die hintere Schulter ( $\triangleright$ Abb. $\mathbf{3 c}$ ) und das Neugeborene gleitet aus dem Geburtskanal.

Während der ganzen Zeit hat die Helferin dabei darauf geachtet, keinen Zug auf das Kind auszuüben und lediglich sanft zu unterstützen.

\section{Fortsetzung folgt}

In der nächsten Ausgabe von retten! führen wir den Fall mit der Versorgung des Neugeborenen fort.

\section{Der Algorithmus}

In der Bundesrepublik werden pro Jahr knapp 700000 Kinder geboren. Nur ein kleiner Teil der Geburten wird außerhalb der Klinik als geplante Hausgeburt oder ungeplante Notgeburt im Rettungsdienst durchgeführt. Legt man diesen Zahlen Erfahrungsdaten aus verschiedenen Rettungsdienstbereichen zugrunde, werden wahrscheinlich ca. 1200 Geburten pro Jahr von Rettungsdienstpersonal begleitet.

Bei Mehrgebärenden ist der Zeitraum zwischen Geburtsbeginn und Austreibungsphase in der Regel sehr viel kürzer als bei Erstgebärenden. Dies wird von den werdenden Müttern mitunter unterschätzt und es kommt viel rascher zur Geburt als erwartet.

\section{Merke}

Die ungeplante Hausgeburt im Rettungsdienst ist ein eher seltener Notfall. Umso wichtiger ist es, sich mit dem Ablauf und den notwendigen Maßnahmen regelmäßig zu beschäftigen.

Das Team im oben beschriebenen Fall nutzt bei der Versorgung der Kreißenden die Muster-Algorithmen des DBRD in der Version 2.0 von 2017.

\section{PRAXIS/MASSNAHMEN}

Laden Sie sich kostenfrei die Algorithmen des Deutschen Berufsverbands Rettungsdienst (DBRD) unter www.dbrd.de herunter.

\section{Transportentscheidung}

Die Entscheidung, ob die Kreißende noch transportiert werden kann oder ob die Geburt vor Ort abgewartet werden sollte, ist für das Rettungsdienstpersonal oft schwierig zu treffen. In der Literatur wird auf die vaginale Beurteilung des Muttermundes verwiesen. Bei einer bevorstehenden Geburt ist der Muttermund $10 \mathrm{~cm}$ weit 
geöffnet - ein Transport sollte dann nicht mehr angegangen werden.

\section{Merke}

Aufgrund der fehlenden Routine des Rettungsdienstpersonals erscheinen die vaginale Inspektion und die Beurteilung des Muttermundes allerdings wenig geeignet. Einfacher ist es, regelmäßige Wehen im Abstand von weniger als 2 Minuten, den Beginn von Pressdrang und Presswehen und das Hervortreten des Anus als Indikatoren für die Entscheidung heranzuziehen.

Nicht vergessen werden sollte in diesem Zusammenhang die Einschätzung der werdenden Mutter. Steht eine Geburt kurz bevor, weiß dies die Mutter oft sehr genau (besonders bei Mehrgebärenden) und kann dies auch klar äußern.

\section{Bevorstehende Geburt}

Im beschriebenen Fall kam es bereits zum Blasensprung und die Frau hat regelmäßige Wehen mit Pressdrang im Abstand von unter 2 Minuten. Da im Mutterpass eine Schädellage beschrieben ist, entscheidet das Team, die Geburt vor Ort abzuwarten, und bereitet dazu alles Notwendige vor. Die Entscheidung, die Geburt im Wohnzimmer auf dem Boden vorzunehmen, hat vor allem wegen des großzügigen Platzes Vorteile: Die Helferin kann sich ohne Probleme im Bereich des Beckens positionieren und so ideal bei der Geburt unterstützen. Der Helfer kann die Geräte und das notwendige Material in unmittelbarer Nähe der Gebärenden bereitstellen.

Das Legen eines peripher venösen Zugangs ist zu diesem Zeitpunkt nicht zwingend notwendig. Da es im Rahmen der Geburt und in der Nachgeburtsperiode jedoch zu Komplikationen kommen kann, sollte ein Zugang zeitnah gelegt werden. Die unterstützenden Maßnahmen bei der Geburt und die anschließende Versorgung des Neugeborenen haben allerdings Vorrang.

\section{Merke}

Hat die Kreißende vor der Geburt nicht abgeführt, ist es typisch, dass mit den Presswehen Stuhl abgeht. Ein wasserundurchlässiges Einmallaken und eine dickere saugstarke Unterlage verhindern ein Verschmutzen des Teppichs und können nach der Geburt einfach entsorgt werden.

\section{Geburt}

Nachdem das Team alle notwendigen Vorbereitungen für die Geburt getroffen hat, unterstützt es die Gebärende in der Austreibungsphase. Im beschriebenen Fall ist die Geburt des Kindes komplikationslos.
PRAXIS/MASSNAHMEN

Sollten Sie in Ihrem Rettungsdienstbereich ein abweichendes Konzept nutzen, strukturieren Sie das beschriebene Fallbeispiel anhand des bei Ihnen eingesetzten Algorithmus und arbeiten Sie die Unterschiede heraus.

\section{Prüfungsfragen}

\section{NOTFALLMEDIZIN}

Beschreiben Sie das für eine Notgeburt und die Versorgung des Neugeborenen notwendige Material. Welches Material benötigen Sie zwingend, und woran sollten Sie außerdem denken?

Für die Geburt des Kindes genügen Einmalhandschuhe für den Helfer sowie eine saugfähige Unterlage. Ideal sind zudem weiche Unterlagen und die Möglichkeit, die Gebärende mit leicht erhöhtem Oberkörper zu lagern.

Wenn möglich, sollte noch vor der Geburt ein venöser Zugang etabliert werden. Zur Überwachung der Vitalparameter sind eine Blutdruckmanschette und ein Stethoskop sowie eine Pulsoxymetrie ausreichend.

Für die Versorgung des Neugeborenen wird ein Orosauger benötigt, mit dem die Atemwege freigemacht werden können. Zwingend ist ein Babybeatmungsbeutel mit Reservoir und Sauerstoff, um bei Bedarf sofort mit der Beatmung beginnen zu können. Für die Abnabelung des Kindes werden zwei Nabelklemmen sowie eine sterile Schere bzw. ein Skalpell benötigt. Zur taktilen Reizung des Neugeborenen ist ein weiches Handtuch oder (wenn nicht vorhanden) ein Verbandtuch sinnvoll. Mit ihm wird das Neugeborene vorsichtig getrocknet. Wird das Kind mit einer sogenannten Glückshaube (geschlossene Fruchtblase) geboren, kann diese am einfachsten mit einer sterilen Klemme eröffnet werden.

Mögliche weitere Fragen:

Welche Informationen erhalten Sie aus dem Mutterpass?

Bei welchen Kindslagen sollten Sie einen Transport in die Klinik anstreben?

Beschreiben Sie in groben Zügen die Geburt und nennen Sie typische Geburtskomplikationen.

\section{KOMMUNIKATION}

Wie könnte sich der Einsatz bei einer strenggläubigen muslimischen Familie verändern? Worauf würden Sie besonders achten? 
Die Geburt eines Kindes ist auch im Islam ein freudiges Ereignis für die ganze Familie. Eine Geburt innerhalb der eigenen vier Wände hat in der Familie durchaus Tradition, wenngleich in der westlichen Gesellschaft die Geburt in der Klinik üblich ist. Begleitet werden die Gebärenden durch erfahrene Frauen aus der Familie und Nachbarschaft. Dass die werdenden Väter bei der Geburt anwesend sind, ist selten und bleibt eher modernen muslimischen Paaren vorbehalten. Die Teilnahme fremder Männer an der Geburtsbegleitung ist nicht üblich und könnte zu Problemen führen.

Wie bei allen Notfallsituationen sollte das Rettungsdienstpersonal durch sein kompetentes und sicheres Auftreten mögliche Fragen gleich zu Beginn mit den Beteiligten klären. Ideal ist es, den Angehörigen die notwendigen Schritte zu erläutern. Durch Hinzuziehen einer oder mehrerer weiblicher Vertrauenspersonen können die Vorbehalte meist gut ausgeräumt werden.

Wenn möglich, ist es für die Frauen einfacher, wenn sie durch eine weibliche Notfallsanitäterin betreut werden. Auch dabei gilt selbstverständlich der Grundsatz, dass das am besten ausgebildete und geburtserfahrenste Personal bei der Geburt unterstützen sollte.

Nach der Geburt und dem Abnabeln sollte der Mutter oder einem Angehörigen die Möglichkeit gegeben werden, dem Neugeborenen nach alter Tradition das Glaubensbekenntnis ins rechte Ohr zu flüstern. Zudem kommt der Nabelschnur im Islam eine besondere Bedeutung zu. Aus diesem Grund sollte diese nicht unbeachtet entsorgt werden. Am besten bespricht man sich darüber nach der Geburt mit dem frischgebackenen Vater.

\section{Mögliche weitere Fragen:}

Was würden Sie im Umgang mit Sprachbarrieren besonders beachten?

Welche Hilfsmittel können helfen, mit dem Patienten nonverbal zu kommunizieren?

Welche Ängste und Sorgen bewegen die werdenden Eltern in dieser Situation? Reflektieren Sie deren Bedürfnisse und Wünsche.

\section{RAHMENBEDINGUNGEN}

Welche organisatorischen Maßnahmen treffen Sie, wenn Sie sich für eine Notgeburt vor Ort entschieden haben? Begründen Sie Ihre Entscheidungen.

Bei einer Notgeburt sollte zum Notarzt in jedem Fall ein zweites Rettungsmittel mit angefordert werden. Ideal wäre ein Fahrzeug mit einem Inkubator, das zudem mit einem Pädiater oder Gynäkologen besetzt ist.
Kommt es während der Geburt zu Komplikationen, sieht sich das Team plötzlich mit zwei Patienten konfrontiert. Sind nicht genügend Helfer und Transportmöglichkeiten vor Ort, kann dies rasch zu einer problematischen Situation führen. Bei Zwillings- oder Mehrlingsgeburten sind entsprechende Kapazitätsanpassungen vorzunehmen. In der Regel empfiehlt es sich in diesen Fällen, die Geburt in die Klinik zu verlagern.

Verläuft die Geburt ohne Schwierigkeiten, werden Mutter und Kind zusammen in einem Fahrzeug transportiert. Damit die Geburt nicht gestört wird, sollte die zweite Besatzung bei unproblematischem Geburtsverlauf im Wartestatus vor dem Haus bleiben.

Mögliche weitere Fragen:

Ist ein Transport von Mutter und Kind in einem RTW zulässig? Begründen Sie Ihre Auffassung.

Welchen Stellenwert hat die Dokumentation im Rettungsdienst?

\section{KOMMENTAR}

von Dr. med. Wolfgang C. G. von Meißner, MHBA Eine Geburt im Rettungsdienst gehört zu den seltenen und zugleich spannendsten Notfalleinsätzen. Oft bestehen Unsicherheiten bei den beteiligten Rettungskräften. Die wenigsten Rettungsassistenten und Notfallsanitäter werden während ihrer Ausbildung die Gelegenheit gehabt haben, bei einer physiologischen Geburt im Kreißsaal dabei gewesen zu sein. Es gibt auch Notärzte, die noch nie eine physiologische Geburt im Krankenhaus miterlebt haben. Dies liegt auch daran, dass in der modernen Geburtshilfe die Geburt so natürlich und intim wie möglich in angenehmer Umgebung für die Eltern stattfinden soll. Immer beliebter werden auch die sogenannten Hebammenkreißsäle. Dort können die Frauen mit Unterstützung einer Hebamme im Krankenhaus ihr Kind auf die Welt bringen. Ärztliche Geburtshelfer werden nur bei Komplikationen hinzugezogen. Auch dabei gilt, dass das Prinzip der Betreuungskontinuität im Geburtsprozess keinen Raum für die Notfallsanitäterausbildung in der Geburtshilfe lässt.

Auch wer selbst schon Kinder auf die Welt gebracht hat oder physiologische Geburten als Vater oder andere(r) Angehörige(r) erleben durfte, sollte sich im Rahmen eines Simulationstrainings mit dem natürlichen Geburtsverlauf und den möglichen Komplikationen beschäftigen.

Grundsätzlich gilt, dass es fast nie ein Fehler ist, die schwangere Frau in den Rettungswagen zu bringen und wenn möglich den Transport in die geburtshiffliche Klinik zu beginnen. Gerade bei vorzeitigem Bla- 
sensprung und noch nicht im Becken festsitzendem Kopf sollte der Transport liegend erfolgen, sonst besteht die Gefahr eines Nabelschnurvorfalls. Geburtsunmögliche Kindslagen müssen unverzüglich ins Krankenhaus transportiert werden. Ein Transport sollte grundsätzlich immer angestrebt werden, wenn der Wehenabstand noch größer als 2 Minuten ist.

Gerade in ländlichen Regionen wurden in den vergangenen Jahren viele geburtshilfliche Abteilungen geschlossen, was zu langen Transportwegen in das nächste geeignete Krankenhaus führen kann. Auch nach der Geburt kann es zu Notfallsituationen kommen; im Fall z. B. einer atonen Nachblutung ist ein schneller Transport in die Klinik für die Mutter lebensrettend.

\section{Interessenkonflikt}

Die Autoren geben an, dass kein Interessenkonflikt besteht.

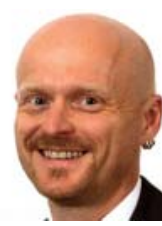

\section{Rico Kuhnke M.A.}

ist Gesamtschulleiter der DRK-Landesschule Baden-Württemberg gGmbH. Er war viele Jahre als Lehrrettungsassistent tätig und hat sein pädagogisches Masterstudium berufsbegleitend abgeschlossen. Er ist Notfallsanitäter und Mitherausgeber von retten!

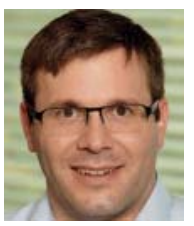

\section{Dr. med. Wolfgang C. G. von Meißner}

ist Facharzt für Anästhesiologie mit den Zusatzbezeichnungen Notfallmedizin und Intensivmedizin. Zugleich ist er Facharzt für Allgemeinmedizin und arbeitet als Landarzt und Notarzt in Baiersbronn im Schwarzwald.

\section{Korrespondenzadresse}

Rico Kuhnke

Schulleiter, Deutsches Rotes Kreuz, Landesschule Baden-

Württemberg

Karl-Berner-Str. 6

72295 Pfalzgrafenweiler

r.kuhnke@drk-Is.de

\section{Bibliografie}

DOI https://doi.org/10.1055/s-0043-119607

retten 2018; 7: 96-103

(c) Georg Thieme Verlag KG, Stuttgart · New York

ISSN 2193-2387 